\title{
Optimization of ECE diagnostics for the W7-X stellarator
}

\author{
N.B. Marushchenko, A. Dinklage, H.J. Hartfuß, M. Hirsch, H. Maaßberg, Yu. Turkin
}

Max-Planck-Institut für Plasmaphysik, EURATOM Association,

Teilinstitut Greifswald, D-17491 Greifswald, Germany

Contact person: Nikolai B. Marushchenko

\author{
Wendelsteinstrasse 1, D-17491 Greifswald, Germany \\ Tel: +49-3834-882330 \\ Fax: +49-3834-882509 \\ e-mail: nikolai.marushchenko@ipp.mpg.de
}

Number of pages: 17

Number of figures: 7 


\title{
Optimization of ECE diagnostics for the W7-X stellarator
}

N.B. Marushchenko*, A. Dinklage, H.J. Hartfuß, M. Hirsch, H. Maaßberg, Yu. Turkin Max-Planck-Institut für Plasmaphysik, EURATOM Association,

Teilinstitut Greifswald, D-17491 Greifswald, Germany

\begin{abstract}
Accuracy and abilities of the ECE diagnostic system planned to be installed in the W7-X stellarator are analysed with help of ray-tracing simulations. For the expected plasma parameters, the spatial resolution of the standard low-field-side (lfs) X2-mode observation scheme is estimated to be sufficiently high, about 5\%. Apart from the $l f s$ scheme, the applicability of other complementary schemes is analysed, in particular, highfield-side ( $h f s$ ) X2-mode observation. It is shown, that, in combination with the standard $l f s$ scheme, the $h f s$ scheme can be very informative for the problem of distinguishing thermal and non-thermal contributions in the ECE spectrum.
\end{abstract}

\footnotetext{
* e-mail nikolai.marushchenko@ipp.mpg.de
} 


\section{INTRODUCTION}

The interpretation of diagnostic data at the W7-X stellarator (under construction in Greifswald, Germany) will be supported by the concept of integrated data analysis $[1,2]$. This concept has been demonstrated to be feasible for the Thomson scattering diagnostic on W7-AS [3]. For the ECE diagnostic, the basic idea is to fit the measured spectral intensity, $I_{\omega}$, simulated by modeling the data (the antenna, the receiver, etc) for a $T_{e}$ profile. However, one needs to estimate (possible) non-thermal contributions in the ECE spectrum, and to find possibilities to extract from measurements the information about these non-thermal effects. Analysis of the capabilities of the ECE diagnostic system, which will be installed at the W7-X stellarator, and a checking of any possible improvements are the main goals of the present work.

The W7-X stellarator is the large-scale device (average major radius $R_{0} \simeq 5.5 \mathrm{~m}$, and plasma radius $a \simeq 0.53 \mathrm{~m}$ ), equipped with superconducting coils, with a low-shear configuration of Helias (Helical Advanced Stellarator) type [4] with five field periods. In Fig. 1, together with the $B$ isolines the flux-surfaces for the so-called "standard" configuration are shown (the left and right frames are referred below as "bean-shaped" plane and "triangular" plane, respectively). While the "bean-shaped" plane corresponds to the maximum $B$ region, the "triangular" plane has the minimum of $B$, and the ratio $B_{\max } / B_{\min }$ on axis can be significantly varied, from 1.0 up to 1.22 for the so-called the "low-mirror" and the "high-mirror" configurations, respectively (for the "standard" configuration $\left.B_{\max } / B_{\min }=1.09\right)$. The corresponding trapped particles fractions on axis are varied from $f_{t r} \simeq 0.02$ for "low-mirror" up to $f_{t r} \simeq 0.45$ for "high-mirror", and $f_{t r} \simeq 0.3$ for the "standard" configuration.

In the initial stage, $\mathrm{W} 7-\mathrm{X}$ will operate in the $10^{19} \mathrm{~m}^{-3}$ range of densities, with strong ECR heating (up to $10 \mathrm{MW}$ ) by X2-mode at $140 \mathrm{GHz}$. The expected electron temperature range (sup- 
ported by transport simulations [5]) is $3-10 \mathrm{keV}$. For a highly localized deposition profile, one has to expect the appearance of supra-thermal electrons. The main launch ports are situated at the low-field side near the "bean-shaped" plane, and the input power must be absorbed mainly by passing electrons. As an option, also the port near the "triangular" plane can be used (currently under discussion), where the power can be launched from the high-field-side (but from the outer side of device). This scenario gives a direct way to produce the supra-thermal electrons and to study their influence on the transport physics $[6,7]$.

The standard scheme of ECE measurements at the 2nd harmonic X-mode is based on i) a good (spatial) localization of the "emission line", which means a high optical thickness of the plasma for an observed radiation and sufficiently high $\nabla B$ along the sightline, and ii) a Maxwellian electron distribution function. While for the appropriate frequency range the first condition is well satisfied (the ECE diagnostic system is planned to be installed near the "bean-shaped" plane $\phi=0^{\circ}$, where $\nabla B$ is largest, see Fig. 1 and Fig. 2), the second one can be violated, especially for low density ECR heated plasmas. For a highly localized deposition profile, one has to expect an appearance of a significant supra-thermal population of electrons. Nevertheless, for more or less typical discharge parameters the bulk electrons are very close to Maxwellian. In the standard lowfield-side (lfs) observation scheme, the main contribution in the emission is usually produced by these bulk electrons with energies of not more than roughly $2 T_{e}$, i.e. the measured ECE spectrum is close to the thermal one.

The detection of non-thermal effects requires a special technique. The attractive idea of using vertical chords with $|B| \simeq$ const as sightlines for the reconstruction of the electron distribution function (see, e.g. Ref. 8) will fail due to the diamagnetic effect (no way to find the chord with $|B| \simeq$ const for an appropriate range of $\beta$-values). Another factor which forces one to take great care in interpretation of vertical ECE data is the possible bending of the radiation 
rays near the resonances due to thermal effects [9], which can be negligible only for very low densities. However, a high-field-side $(h f s)$ observation (especially along the same sightline as the Ifs one [10]) appears to be much more promising: despite the moderate spatial resolution of a $h f s$ ECE diagnostic, the existence of supra-thermal electrons can be identified by comparison of both Ifs and $h f s$ ECE spectra. Abilities of a $h f s$ diagnostic as a tool to indicate the emission of suprathermal electrons in tokamaks have been examined both theoretically and experimentally [11-13], where the high sensibility of the non-thermal ECE is confirmed. But the interpretation of the $h f s$ measured ECE spectra is not trivial and requires special attention.

Apart from the $l f s$ and $h f s$ observations near the "bean-shaped" plane, the use of the "triangular" plane $\left(\phi=36^{\circ}\right.$, near the minimum of $\left.|B|\right)$ is also under discussion. Here, $|B|$ decreases along the sightline (from outside to inside) similar to the $h f s$ case near the $\phi=0$ plane discussed above. An additional advantage of the "triangular" plane for ECE measurements is the possibility to identify the trapped electron's contribution in ECE, which is much smaller (or even absent) near the "bean-shaped" plane, where the maximum of $B$ is located.

\section{RESULTS OF RAY-TRACING SIMULATIONS}

The ECE spectrum and its spatial resolution are calculated by a newly developed ray-tracing code, which operates with magnetic field configurations obtained from VMEC [14] calculations. For the simulations, the so-called W7-X "standard" vacuum configuration was used. The absorption coefficient and the emissivity are defined in the general formulation (see, e.g. Ref. 15), by the anti-Hermitian part of the dielectric tensor and the micro-current correlation tensor, respectively. Both these tensors are calculated by integration of the arbitrary fully relativistic electron distribution function. In the code, the contributions of the (ripple-) trapped and passing electrons are calculated separately. The last tool is very useful for stellarator-specific problems, where the 
different classes of electrons have to be taken into account. The polarization vectors and the power flux are defined with the traditional slightly relativistic (small Larmor radius approach) dielectric tensor. This model is quite general and covers completely the relevant parameter range.

The electron radiative temperature, $T_{\text {ece }}$, for a given frequency, $\omega=2 \pi f$, is obtained from the radiative transport equation

$$
T_{e c e}(\omega)=\left\langle\frac{8 \pi^{3} c^{2}}{\omega^{2}} g(\omega) \sum_{\text {rays }} w_{\text {ray }} \int_{s_{0}}^{s_{1}} \mathrm{~d} s^{\prime} \eta_{\omega}\left(s^{\prime}\right) \mathrm{e}^{-\left(\tau_{\omega}\left(s_{1}\right)-\tau_{\omega}\left(s^{\prime}\right)\right)}\right\rangle_{\omega \pm \Delta \omega / 2}
$$

where $\eta_{\omega}$ and $\tau_{\omega}$ are emissivity and optical depth, respectively. The antenna beam is discretized by the number of rays, and the ray weight factor, $w_{\text {ray }} \propto \mathrm{e}^{-\rho_{\text {ray }}^{2} / 2 b^{2}}$ (here, $\rho$ is the ray radius, and $b$ is the beam cross-section size), being the antenna beam Gaussian pattern, describes the radiation intensity distribution in the beam. For each frequency, $\omega$, the results are averaged over the $\omega \pm \Delta \omega / 2$ range with the correspondent frequency band function, $g(\omega)$, which is the radiometer characteristic (in the present simulations it has been assumed rectangular for each frequency channel, i.e. $g(\omega)=1 / \Delta \omega$ for $\omega \in \omega \pm \Delta \omega / 2)$. Multiple reflections are not taken into account since it is expected that the torus will be covered by $\mathrm{B}_{4} \mathrm{C}$ to strongly increase the wall absorption and to decrease ECRH stray radiation. Thus, the reflection from the inner wall is assumed to be negligible in all calculations.

The problem of "mapping" the ECE spectrum onto the proper magnetic surfaces also requests special attention. Due to the relativistic (and Doppler ) broadening, a non-locality of the emission line arises, when the gradients of density and temperature can also be important. Instead of the "cold" resonance position, $\mathbf{X}_{c y}$ for a given frequency, one needs to find the "weighted" center of the emission line, $\mathbf{X}_{e c e}$, and to estimate its spatial width, $\Delta X_{e c e}$, (i.e. the spatial resolution). For the calculations, the same algorithm as in Ref. 16 is used: the point at the sightline, where half 
of the integral emission intensity is reached, is called the center of the (asymmetric) emission line. Including in the integral emission intensity only $90 \%$ of the total value, the respective width of the emission line is estimated by "cutting off the rest of its wings". As the final step, the "weighted" center of the emission line, $\mathbf{X}_{e c e}$, together with its wings are mapped onto the magnetic coordinates, producing $r_{\text {eff }}\left(\mathbf{X}_{e c e}\right)$ and the appropriate "error bars". The approach for calculating the emissivity and reabsorption (integration along the resonance curve in momentum space) gives the possibility to estimate also the velocity range of electrons, which produce the main contribution to the measured emission.

The aim of this work is to estimate the contribution of the supra-thermal electrons in ECE. In principle, in order to study this effect in details, Fokker-Planck simulations are required. Nevertheless, for preliminary estimations of the non-thermal contributions in the ECE spectrum (without looking to any concrete scenario), it is sufficient to use the simplified bi-Maxwellian model. The electron distribution function is represented as $f_{e}=(1-\delta) f_{0}+\delta f_{1}$, with $f_{0}$ and $f_{1}$ being Maxwellian distribution functions, the main one and the supra-thermal one, respectively. For the highly localized ECRH deposition profile, it is also assumed, that the supra-thermal fraction exists only near the axis, $r_{\text {eff }} \lesssim \Delta=5 \mathrm{~cm}$, with a Gaussian shape of its weight, $\delta=\delta_{0} \mathrm{e}^{-r_{\text {eff }}^{2} / \Delta^{2}}$. Radial dependence of the supra-thermal fraction temperature is defined in a similiar way, $T_{e 1} / T_{e 0}=1-\mathrm{e}^{-r_{\text {eff }}^{2} / \Delta^{2}}\left(1-T_{e 1}(0) / T_{e 0}(0)\right)$. In this paper, the supra-thermal fraction is assumed as $\delta_{0}=0.05$ and $T_{e 1}(0) / T_{e 0}(0)=3$, which corresponds to $15 \%$ of the energy contained in supra-thermal electrons in the plasma center. Despite its crudeness, this model is more or less adequate to the expected quasi-linear flattening of the electron distribution function, producing a non-negligible supra-thermal fraction in the most interesting range, $v / v_{t h} \approx 1.5-3$ with $v_{t h}=\left(2 T_{e} / m\right)^{1 / 2}$. (Note, that usually, excluding exotic conditions, the more high-energy tails, $v / v_{t h}>4$, are not so important due to their negligible contribution in the ECE spectrum.) 
Simulations are performed for $n_{e}(0)=2 \cdot 10^{19} \mathrm{~m}^{-3}$ with an almost flat profile near the axis (within the heated region), and for a peaked $T_{e}$ profile with $T_{e}(0)=5 \mathrm{keV}$. The magnetic field on axis at the "bean-shaped" plane, $\phi=0$, is taken to be $B=2.5 \mathrm{~T}$.

The port for the main lfs ECE antenna (see Fig. 2) is situated at $R_{l}=6.5 \mathrm{~m}, \phi_{l}=6.1^{\circ}$ (near the "bean-shaped" plane), and $Z_{l}=0.35 \mathrm{~m}$ (somewhat above the equatorial plane). It is assumed, that the antenna has a thin cone of divergence, taken equal to $1.2^{\circ}$, and the (beam) crosssection size at the antenna (initial beam waist) is $b=1 \mathrm{~cm}$. All frequency channels are assumed to have the same band width of $\Delta f=0.35 \mathrm{GHz}$, and the results are averaged over $f \pm \Delta f / 2$. The direction of observation is defined by the poloidal angle, $\vartheta$, which is the deviation from the $R-\phi$-plane, and the toroidal angle, $\varphi$, which is the deviation from the $R-Z$-plane, where the line with $\vartheta=0$ and $\varphi=0$ is directed to the center of the device $(R=0)$. The poloidal and toroidal angles of the $l f_{s}$ observation $\left(\vartheta_{l}=-17^{\circ}\right.$ and $\varphi_{l}=-5^{\circ}$, respectively) are chosen i) to see the axis, and, ii) to minimize the values of $N_{\|}$along the sightline, keeping, in particular, $N_{\|}\left(r_{\text {eff }}=0\right) \simeq 0$ (for the central ray, at least). The $h f s$ antenna is assumed to be located at the opposite position, $R_{h}=5.58 \mathrm{~m}, \phi_{h}=5.3^{\circ}, Z_{h}=0.05 \mathrm{~m}$, with the direction $\vartheta_{h}=163^{\circ}$ and $\varphi_{h}=-5^{\circ}$. For operation at moderate densities, the refraction effects are almost negligible, and both sightlines should coincide. For the chosen sightline, mainly the passing electrons are seen by the ECE antenna, and the total fraction of trapped electrons contributing to the ECE is less than $15 \%$.

For $B=2.5 \mathrm{~T}$ on axis at $\phi=0^{\circ}$, the magnetic field varies along the ECE observation chord between $2.25 \mathrm{~T}$ and $2.82 \mathrm{~T}$, and the resonance frequencies for the neighbouring harmonics (2nd and 3rd) have no overlap (from $126 \mathrm{GHz}$ to $158 \mathrm{GHz}$ and from $189 \mathrm{GHz}$ to $237 \mathrm{GHz}$, respectively). The frequency range from $115 \mathrm{GHz}$ to $220 \mathrm{GHz}$, used for the simulations, covers both the 2nd and the 3rd harmonics ranges. Note, that the upper and lower frequency limits are not the same as the 
"cold" resonance limits. The lower frequency limit (2nd harmonic) is chosen to cover the downshifted emission from the supra-thermal electrons for both $l f s$ and $h f s$ cases. The 3rd harmonic range is discussed below. For comparison, in Fig. 3 both the $l f s$ and the $h f s$ ECE spectra are shown. One can see, that the spectrums obtained for the neighbouring harmonics (2nd and 3rd) are indeed well separated.

Let us first analyse the range of the 2nd harmonic. Excluding the low-frequency range ( $f<127 \mathrm{GHz}$ ), for which the plasma is optically thin, the $l f_{s}$ spectrum does not show any visible difference between the thermal and the non-thermal spectrum. Because of the high optical depth, the "weighted" center of the emission line for each frequency (127 GHz $\leq f \leq 157 \mathrm{GHz})$ has a very small shift from the "cold" resonance $\left(\omega=2 \omega_{c e}\right)$ position. However, the $h f s$ spectrum has a more pronounced peak at low frequencies, indicating the contribution of the supra-thermal electrons. Despite the fact, that for these frequencies the "cold" resonance position, $\omega=2 \omega_{c e}$, is far from the axis (and may be even outside of the plasma), the "weighted" center of the emission line, being observed from $h f s$, is located near the axis. This is clearly seen in Fig. 4, where the spatial emissivity profiles at $f=128 \mathrm{GHz}$ for both cases are shown, without (upper figure) and with (lower figure) reabsorption. The chosen frequency corresponds to the top of the $h f s$ spectrum. One can see, that due to strong reabsorption, the emission observed from the $l f s$ is coming from the region very close to the "cold" resonance position, $R \simeq 6.08 \mathrm{~m}$, i.e. from the periphery of the plasma. And, vice versa, emission observed by the $h f s$ antenna, originates from the central region of the plasma, $R \simeq 5.9 \mathrm{~m}$, where the non-thermal population exists. Furthermore, while the cyclotron emission, being the local characteristic, does not have any dependence on the observation direction, the energy range of electrons, which are responsible for the reabsorption, is defined exactly by the direction of observation, and it is quite different for the $l f s$ and $h f s$ cases. In other words, the down-shifted emission of energetic electrons near the axis, which propagates 
in the direction of increasing $B$ is not reabsorbed, and can be identified in the measurements. The importance of this difference between the $l f s$ and $h f_{s}$ spectra for the interpretation and for the "mapping" of it onto the radius, is demonstrated in Fig. 5. Here are shown the major radius projections of the weighted center of the emission line, $R_{e c e}$, versus the corresponding "cold" resonance (major radius) position, $R_{c y}$. As expected for the lfs spectrum, $R_{e c e}$ is very close to $R_{c y}$, being down-shifted by not more than $2 \mathrm{~cm}$. But the $h f s$ spectrum has $R_{e c e}$ strongly shifted below $R_{c y}$ inside the plasma. In fact, the spatial location of the emission line is dominated by reabsorption, and not by emission.

In principle, the low-frequency part of the $l f s$ spectrum $(f<127 \mathrm{GHz})$ also contains the information about the supra-thermal electrons from the central region (see Fig. 3). Nevertheless, due to non-locality (for these frequencies the plasma is optically thin) it is almost impossible to distinguish the emission from the periphery and the central regions of the plasma. For confirmation, we refer to the ECRH experiments at W7-AS [6], where the described feature (low frequency "hump") was observed. The main conclusion there was that these results cannot be simply interpreted as the emission by suprathermal electrons located only within the narrow deposition profile. The results were very sensitive to the scenario, and this low frequency "hump" was most pronounced in the case of X2-mode heating with a minimum of $B$ in the launching plane, when a significant contribution to the radial transport was produced by the ECRH-driven convection [7].

One can compare also the velocities of electrons, which contribute to the emission (Fig. 6, left). Due to the strong reabsorption, the bulk electrons with $v / v_{t h} \simeq 1$ are responsible for the lfs ECE spectrum. But for the $h f s$ ECE spectrum, much more energetic electrons contribute to the EC emission, especially in the range $115 \mathrm{GHz} \leq f \leq 130 \mathrm{GHz}$, where only (supra-thermal) tails of the distribution function with $v / v_{t h} \sim 3-4$ are responsible. Note, that the difference in electron energies for the $l f s$ and the $h f s$ ECE spectra is quite large for almost all frequencies. One can see 
also, that the velocity range of the $l f s$ spectra for $f \leq 127 \mathrm{GHz}$ almost coincides with the $h f s$ one, having $v / v_{t h} \sim 3-4$. As was already discussed above, the reason is that the periphery plasma is optically thin for the downshifted 2nd harmonic emission from the (fast) electrons coming in lfs direction from the plasma center.

For another scenario with off-axis non-Maxwellian electrons, the comparison of $h f s$ and $l f s$ should be a powerful tool. In Fig. 6 (right), the velocities of emitting electrons are shown, mapped onto the effective radius (procedure of mapping is described above). Here is clearly seen that the low frequency channels of the $h f s$ observation contain only the information from the central region of the plasma. The $h f s$ measurements do not reproduce the "right hand" part of the $T_{\text {ece }}\left(r_{\text {eff }}\right)$ profile, the region $r_{\text {eff }}>0$ is covered only by the $l f s$ observation (see also Fig. 5). Observe also in Fig. 6 (right), that the velocity range of emitting electrons is not symmetric in $r_{\text {eff }}$ for both $l f s$ (not strongly pronounced) and $h f s$ cases. For the $l f s$ case, the main reason is that the sightline cannot be perpendicular to the magnetic surfaces everywhere. The value of $N_{\|}$is minimized near the axis, and one can recognize it by a higher localization (in velocity space) of emission coming from $r_{\text {eff }} \simeq 0$. In the other regions the Doppler-broadening becomes important. Because the values of $N_{\|}$and $\nabla B$ are changing along the sightline, an asymmetry of the "left" and the "right" parts of ECE spectrum appears even for the lfs observation. Also the finite width of the beam and the finite $\Delta \omega$ (both are taken into account) play a non-negligible role.

In Fig. 4 are also shown the other part of the ECE spectrum, related to the 3rd harmonic, $180 \mathrm{GHz} \leq f \leq 220 \mathrm{GHz}$. Note, that these frequency limits differ from the appropriate "cold" resonance harmonic range. For this frequency range, the optical depth is much smaller, $\tau \simeq 1$, compared to the 2 nd harmonic, and the plasma is optically gray. As consequence, for the lfs observation, due to the down-shifted emission from the central plasma for the frequencies that correspond to the low-field-side of the plasma $\left(r_{\text {eff }}>0\right)$, and, vice versa, due to the contribution 
of the emission from the high-field-side periphery $\left(r_{\text {eff }}<0\right)$ for the frequencies that correspond to the central region, the 3rd harmonic ECE spectrum is significantly down-shifted. Nevertheless, despite the circumstance that 3rd harmonic measurements do not have sufficient spatial resolution, it has diagnostic potential. First of all, both the $l f s$ and the $h f s$ observations give almost the same spectra (contrary to the 2nd harmonic case, they have almost the same range of velocities for emitting electrons, $v / v_{t h} \sim 1.5-2$ ), and they can be used for verification of the 2 nd harmonic measurements. In principle, exactly this range of energies is expected to be influenced by ECRH, but a clear indication of any disturbance of the distribution function requires special attention. Note, that the non-thermal disturbance (bi-Maxwellian model), used for the simulations, does not produce any noticeable effect in the 3rd harmonic frequency range. Another interesting application of the 3rd harmonic frequency range is the estimation of the inner wall reflection coefficient, which can be done by comparison of the optically thick 2nd harmonic and optically thin 3rd one $[17,18]$.

The ECE diagnostic observation with (almost) perpendicular sightlines does not contain any information on the electron current. In that sense it should be interesting to analyse also the abilities of an oblique observation. This method was discussed also in [19], and it was estimated to be somewhat promising. For W7-X there is the possibility (under discussion now) to use anglescanning of the $l f s$ antenna (the $h f s$ antenna does not have this possibility). In order to model this option, the ECE spectrum has to be calculated for a plasma with an asymmetric electron distribution function, obtained by Fokker-Planck simulations. (The necessary Fokker-Planck simulations will be performed later.) Nevertheless, some preliminary conclusions, related to the velocity range of emitting electrons, can be obtained. First, for any ECCD scenario the bulk electrons $\left(v / v_{t h} \lesssim 1\right)$ are expected to be close to the Maxwellian. In order to distinguish the difference between the opposite angle observations, the radiation has to be emitted by electrons from the tails of the distribution function, othervise, it should be very difficult to interpret the measurements. The results 
of the simulations for two oblique sightlines with the angles $\varphi=\varphi_{l} \pm 10^{\circ}$ are as follows: while the main energy of emitting electrons is not changed much in comparison with quasi-perpendicular observation, $v / v_{t h} \simeq 1.5$, the Doppler broadening becomes rather large, $1.5 \lesssim \Delta v / v_{t h} \lesssim 2$ (compare with $\Delta v / v_{t h} \lesssim 0.5$ for quasi-perpendicular observation near the axis). And, second, because of the non-planar (3D winding) magnetic axis the results of oblique observation must be very sensitive to $\beta$-effects (Shafranov-shift).

Another promising possibility is the ECE observation in the "triangular" plane, $\phi=36^{\circ}$, with the inverted $\nabla B$ along the sightline. The main advantage is that this $h f s$ observation can be performed using the outer port. The position of the ECE antenna is chosen to have a monotonic decrease of $B$ along the sightline $\left(R_{t}=6.5 \mathrm{~m}, \phi_{t}=36^{\circ}, Z_{t}=0.35 \mathrm{~m}\right)$ with minimized $N_{\|}$ $\left(\vartheta_{t}=-16^{\circ}\right.$ and $\left.\varphi_{t}=-4^{\circ}\right)$. The ECE spectrum is qualitatively the same as for the $h f s$ in the "bean-shaped" plane, with down-shifted frequencies corresponding to the lower $B$. In Fig. 7, $T_{\text {ece }}$ and the velocities of emitting electrons for the 2nd harmonic frequency range are shown versus the effective radius. For reference, also the $T_{e}$ profile used for the simulations is given. Similar to the $h f s$ in the "bean-shaped" plane, only one half of the $T_{\text {ece }}\left(r_{\text {eff }}\right)$ profile can be covered by the ECE spectrum, because the low-frequency channels $(110$ - $115 \mathrm{GHz})$ correspond to radiation from the central region. Because of lower $|\nabla B|$, the spatial resolution in the central region is fairly low. Nevertheless, these measurements, having a large contribution of trapped electrons (about $50 \%$ ), should be very informative, especially for investigations of the (optional) ECCD scenario with launching close to the "triangular" plane. In this scenario (the oblique launch of RF power from the $h f s$ ) the power is absorbed by high energetic electrons, and the $h f s$ ECE measurements, respectively, can give the requested information. 


\section{SUMMARY}

As expected, the standard ECE technique, i.e. the lfs ECE measurements near the "beanshaped" plane with 2nd harmonic X-mode, is the most accurate and convenient method to obtain the electron temperature profile. For the integrated data analysis this scheme is applicable due to its sufficiently high spatial resolution (the largest width of the emission line is about $2 \mathrm{~cm}$, i.e. the spatial resolution is about $5 \%$ ).

Additional information, related to non-thermal electrons, can be obtained with complementary $h f s$ measurements in both the "bean shaped" $(\phi=0)$ and the "triangular" $\left(\phi=36^{\circ}\right)$ planes. Apart from simplicity in realization, the "triangular" plane is especially interesting due to the circumstance, that the ripple-trapped electron contribution in the ECE radiation can significantly exceed that from the "bean-shaped" plane. For comparison, in the "triangular" plane of the "standard" configuration the trapped electrons contribution in ECE is about 50\%, while near the "beanshaped" plane it does not exceed 15\%. Despite lower spatial resolution, the $h f s$ spectrum (especially its low-frequncy range) contains interpretable information on the non-thermal population of electrons. It has to be stressed also, that the $h f s$ measurements cannot be interpreted without comparison with the standard $l f s$ results.

The ability of an oblique $l f s$ observation to indicate an asymmetry of the electron distribution function (the electron current) requires additional investigations, based on higher accuracy in modelling of the kinetic effects. The results of the present simulations are not sufficient to give a final answer.

The 3rd harmonic frequency range might provide sufficient information for all schemes discussed above. Despite the low optical depth, $\tau \simeq 1$, the part of the ECE spectrum with $\omega>3 \omega_{c e}(0)$ has a spatial resolution very similar to the 2 nd harmonic $h f s$ spectrum, i.e. $T_{\text {ece }}$ 
is much less than for 2 nd harmonic $l f s$ spectrum, but quite suitable for the identification of the emitting region.

\section{ACKNOWLEDGMENTS}

We wish to acknowledge the support of and fruitful discussions with the W7-X Team and ECRH group. We also gratefully acknowledge the referees (especially the first one) for their careful reading of the manuscript and their helpful criticism. 
[1] A. DINKLAGE, R. FISCHER, and J. SVENSSON, Fusion Sci. Technol. 46, 355 (2004).

[2] R. FISCHER and A. DINKLAGE, Rev. Sci. Instrum. 75, 4237 (2004).

[3] R. FISCHER, A. DINKLAGE, and E. PASCH, Plasma Phys. Control. Fusion 45, 1095 (2003).

[4] J. NÜRENBERG and R. ZILLE, Phys. Letters 114A, 129 (1986).

[5] M. ROMÉ, V. ERCKMANN, U. GASPARINO, and N. KARULIN, Plasma Phys. Control. Fusion 40, 511 (1998)

[6] M. ROMÉ et al., Plasma Phys. Control. Fusion 39, 117 (1997).

[7] S. MURAKAMI et al., Nuclear Fusion 40, 693 (2000).

[8] D.R. ROBERTS et al., Rev. Sci. Instrum. 66, 427 (1995).

[9] E. WESTERHOF, Proc. of the 10th Joint Workshop on Electron Cyclotron Emission and Electron Cyclotron Heating (World Scientific Publishing Co. Pte. Ltd., Ameland, The Netherlands, 1997), p. 39.

[10] N.B. MARUSHCHENKO et al., Proc. of the 31st EPS Conference Plasma Phys., London, 28 June 2 July 2004 ECA Vol.28G P-1.204.

[11] G. GIRUZZI, Nuclear Fusion 28, 1413 (1988).

[12] J.F.M. VAN GELDER, E. WESTERHOF, F.C. SHÜLLER, and A.J.H. DONNE, Plasma Phys. Control. Fusion 40, 1185 (1998).

[13] P. BLANCHARD et al., Plasma Phys. Control. Fusion 44, 2231 (2002).

[14] S. HIRSHMAN and J. WHITSON, Phys. Fluids 26, 3553 (1983).

[15] M. BORNATICI and F. ENGELMANN, Phys. Plasmas 1, 189 (1994).

[16] V. TRIBALDOS and B.P. VAN MILLIGEN, Nuclear Fusion 38, 283 (1996). 
[17] A. KRÄMER-FLECKEN, G. WAIDMANN, and P.C. DE VRIES, Proc. of the 10th Joint Workshop on Electron Cyclotron Emission and Electron Cyclotron Heating (World Scientific Publishing Co. Pte. Ltd., Ameland, The Netherlands, 1997), p. 209.

[18] V.S. UDINTSEV, M.J. VAN DE POL, and A.J.H. DONNÉ, Rev. Sci. Instrum. 72, 359 (2001).

[19] E. DE LA LUNA et al., Rev. Sci. Instrum. 74, 1414 (2003). 
FIG. 1. For one half-period of W7-X, the different cross-sections are shown. The left $(\phi=$ $\left.0^{\circ}\right)$ is called the "bean-shaped" plane, the right $\left(\phi=36^{\circ}\right)$ the "triangular" plane. Along with the magnetic surfaces, the $B$-isolines are also plotted. Note, that the "bean-shaped" plane has a tokamak-like behaviour of $\nabla B$, while the "triangular" plane has an inverted one.

FIG. 2. The cross-section near the "bean-shaped" plane $\left(\phi=6.1^{\circ}\right)$ with the sightline of the main ECE antenna (lfs observations) is shown. The position for the $h f s$ antenna is also marked.

FIG. 3. Wide range ECE spectrum (radiative temperature, $T_{e c e}$ ) for $l f s$ (left) and the same for $h f s$ (right) observations; $\Delta$ - thermal (Maxwellian) spectrum, $\circ$ - non-thermal (bi-Maxwellian) spectrum. Note, that the frequencies of less than $127 \mathrm{GHz}$ correspond to the (cold) resonance positions outside of the plasma.

FIG. 4. Top: Spatial ECE emissivity profiles at $128 \mathrm{GHz}$ (projection to the major radius) for both Maxwellian, $\eta_{M}$, and for bi-Maxwellian, $\eta_{b M}$, are shown. Bottom: The ECE emission line profiles (i.e. emissivity with reabsorption taken into account) for $l f s$ and $h f s$ observations are shown. Note, that for the $l f s$ case the Maxwellian and the bi-Maxwellian emission line profiles are indistiguishable and they both have the same label.

FIG. 5. The "weighted" center of the emission line $v s$ the "cold" resonance position $\left(\omega=2 \omega_{c e}\right)$, projected to the major radius, is shown for both the lfs $(\Delta)$ and the $h f_{s}(\circ)$ cases.

FIG. 6. Velocity ranges (with $v_{t h}=\left(2 T_{e} / m\right)^{1 / 2}$ ) of emitting electrons as function of frequency (left) and effective radius (right) for the "bean-shaped" plane. The markers correspond to the center of the local emission function, where half of the emissivity is obtained. The bars indicate the main velocity range of contributing electrons. Note, that the low-frequency range emission belongs to the central region.

FIG. 7. The radiative temperature (left) and the velocities of emitting electrons (right) $v s$ the effective radius (right) for the "triangular" plane. The solid line shows the $T_{e}$ profile for reference. 

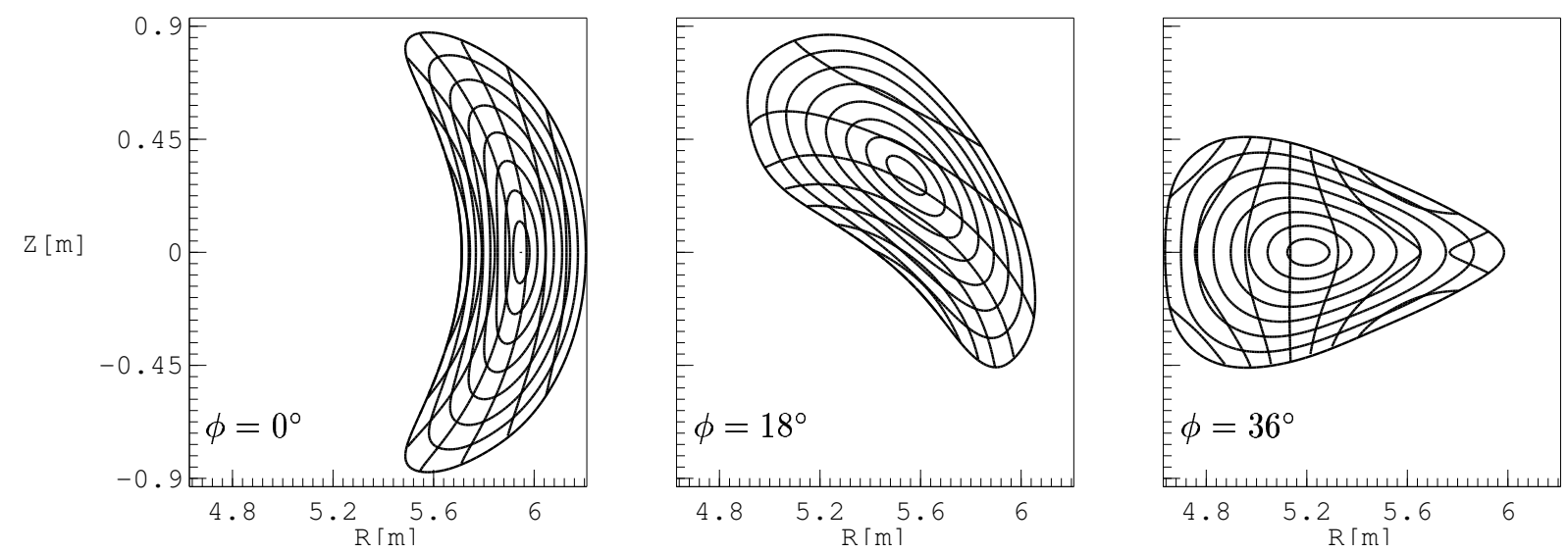

FIG.1. 


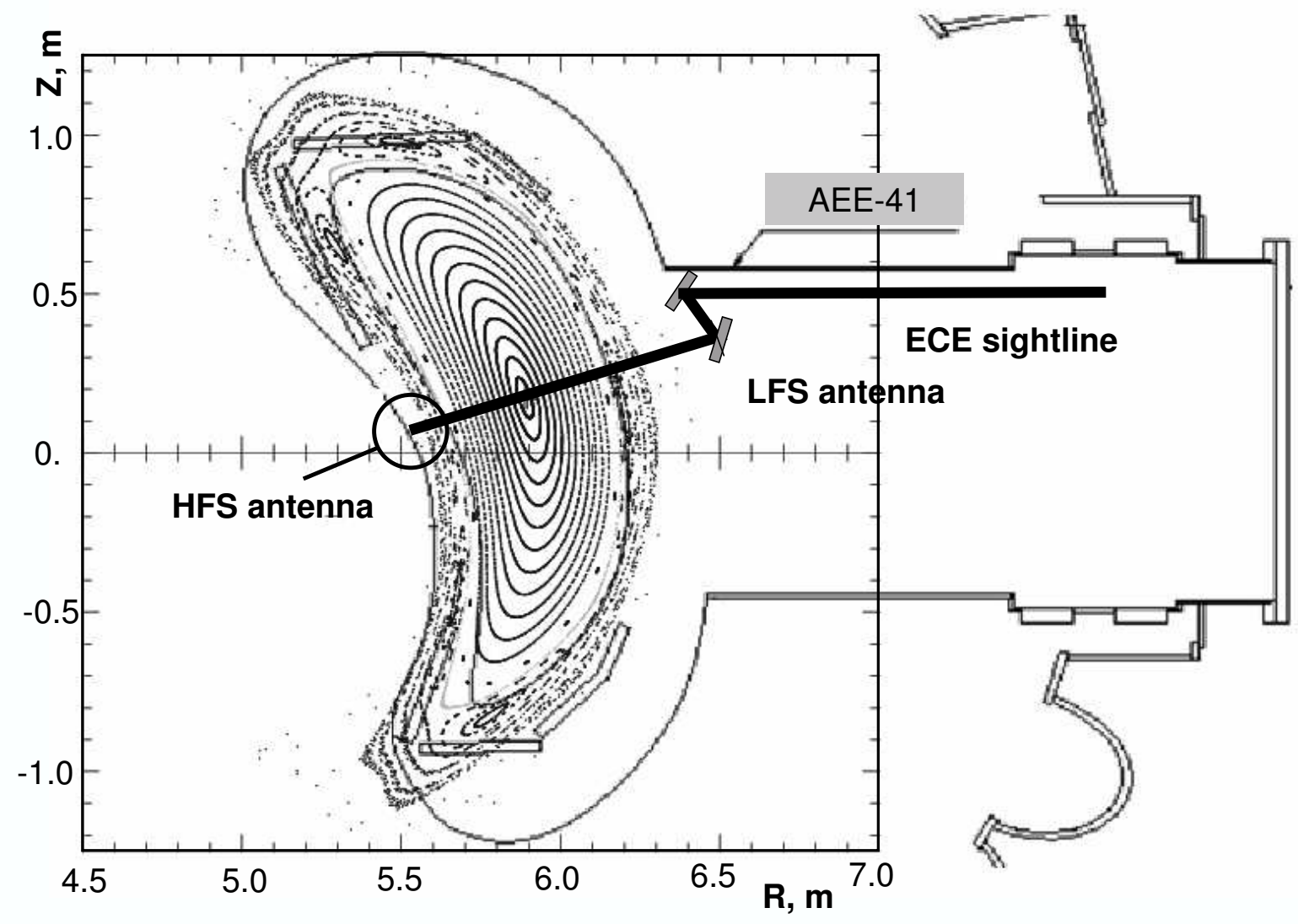

FIG.2 . 

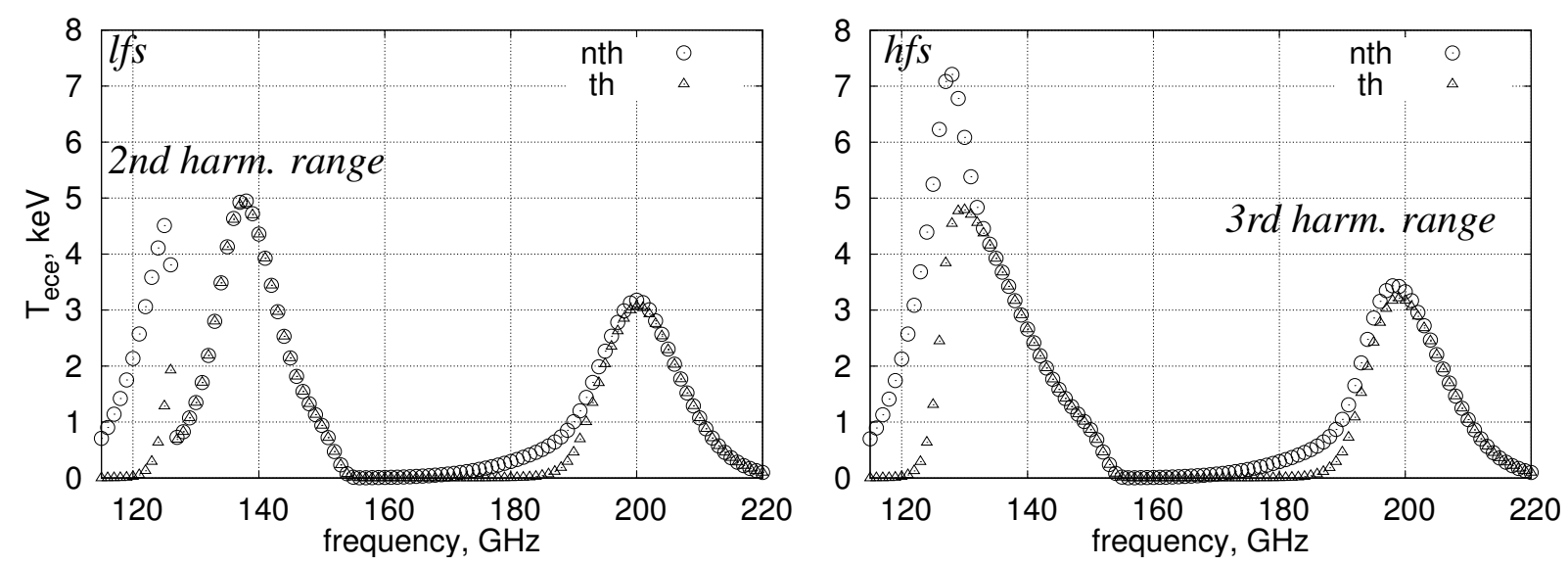

FIG.3. 

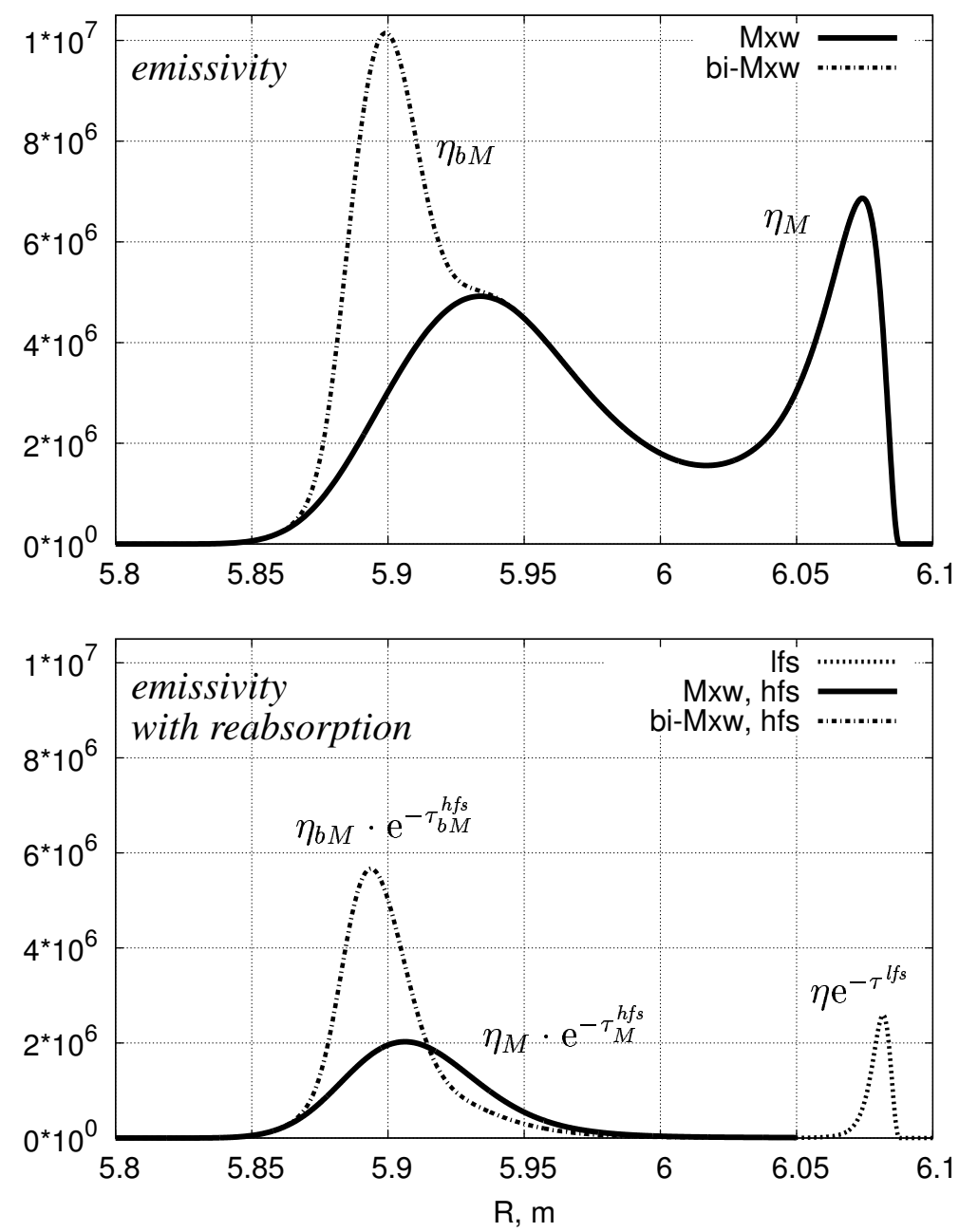

FIG.4. 


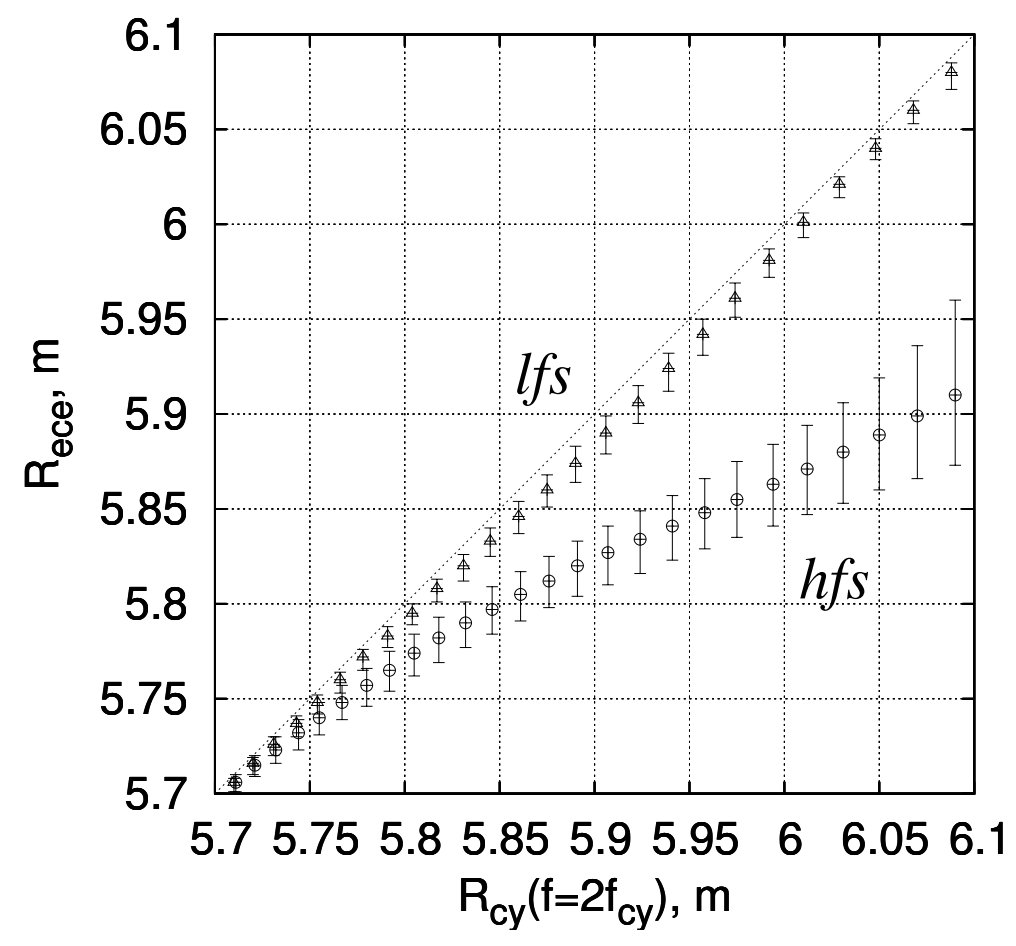

FIG.5. 

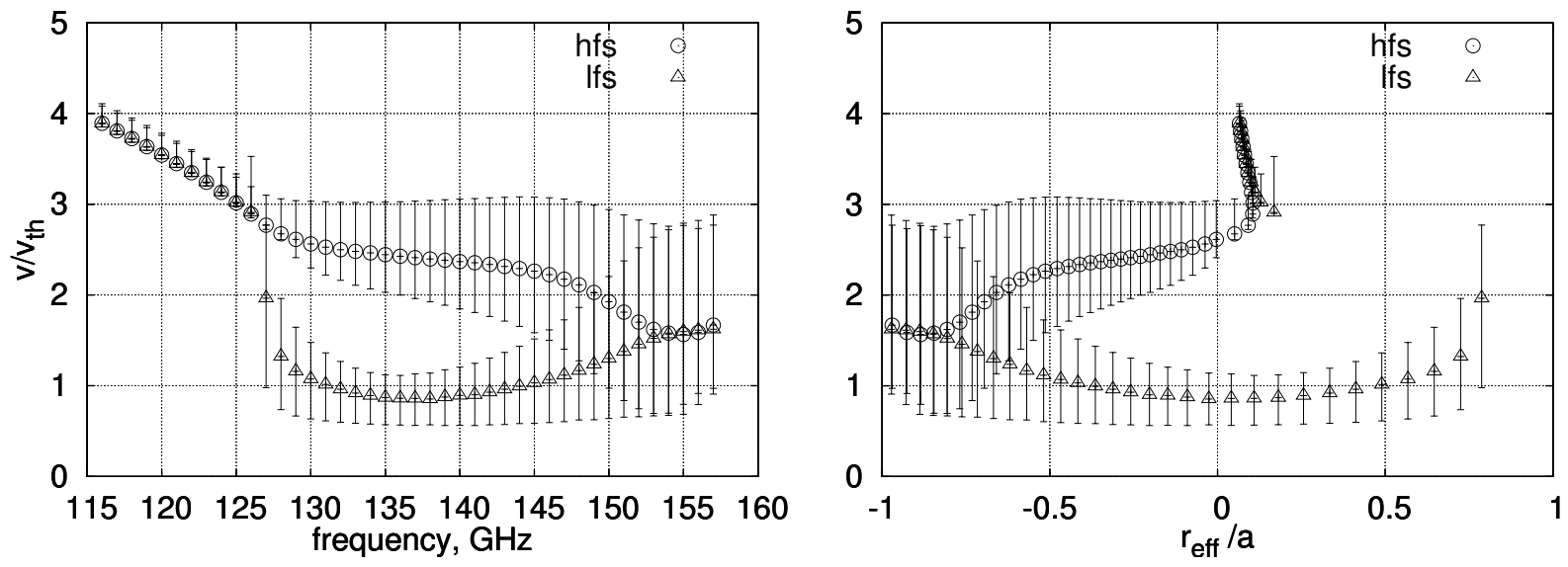

FIG.6. 

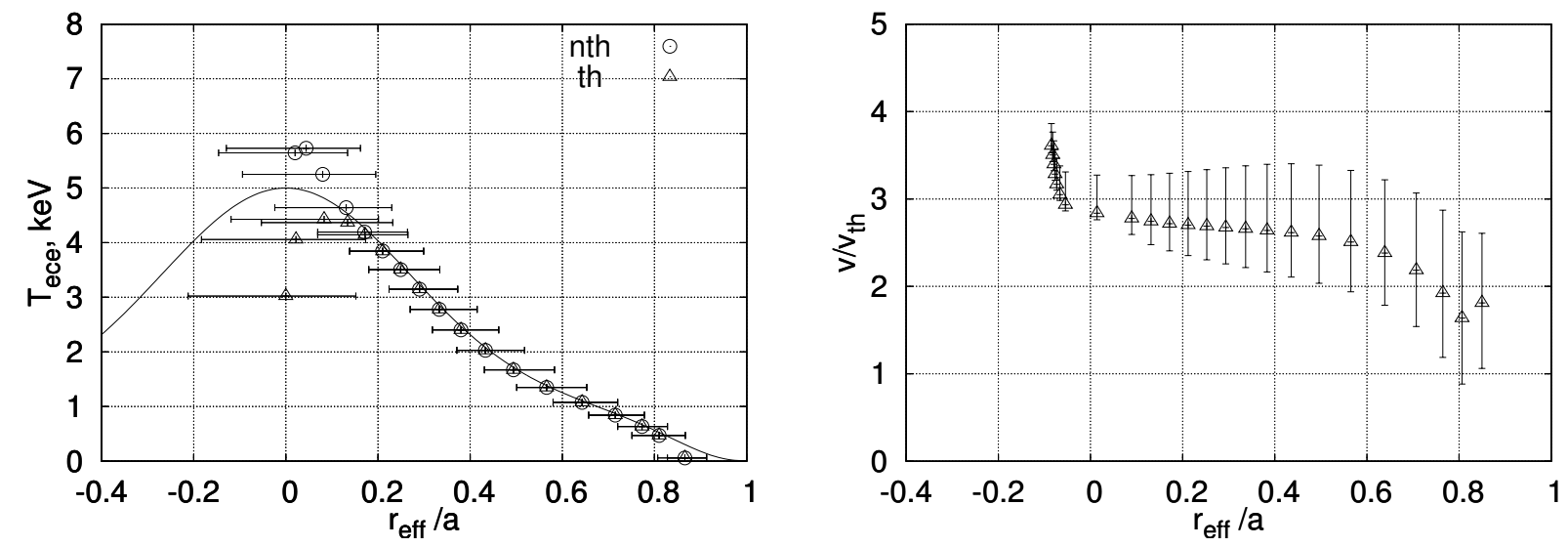

FIG.7. 
Nikolai B. Marushchenko (Dipl, 1980, Kharkov State University, Ukraine; PhD, 1990, plasma physics, Kharkov Institute of Physics and Technology, Ukraine) since 2000 is a scientist in the theory group of Experimental Division E3 at the Max-Planck-Institut für Plasmaphysik (IPP), Greifswald, Germany, where he is responsible for the numerical simulations and the theoretical support of ECRH/ECCD experiments, as well as the ECE diagnostic.

Andreas Dinklage recieved his $\mathrm{PhD}$ in experimental plasma physics from Ruhr-Universität Bochum in 1996 and his habilitation from Greifswald University in 2001. He is responsible for diagnostics software preparation for Wendelstein 7-X and he is project leader in the Transregional Cooperation Centre 24 funded by the German Science Foundation. His research interests cover data analysis in plasma physics, design of diagnostics and confinement in stellarators.

Hans J. Hartfuß graduated from Giessen University where he recieved his $\mathrm{PhD}$ in spectroscopy in 1975. He has been with the Max-Planck-Society since 1978, first in astrophysics and since 1982 in plasma physics. He is head of the project "Diagnostics for W7-X" and honorary professor at the University of Greifswald. Major interests are the development of microwave diagnostic techniques, plasma transport and turbulence studies.

Matthias Hirsch, studied at Univ. of Würzburg, Diploma work on Spectroscopy in crystals 1986, PhD 1991 on Raman Spectroscopy in Semiconductor Structures, Postdoc at IPP developing Reflectometry, currently: group leader topical of the group for microwave diagnostics, research on physics of edge transport barriers and turbulence.

Henning Maaßberg, (Diploma, physics, Technical University Braunschweig, Germany, 1975; PhD, physics, Technical University Darmstadt, Germany, 1980) has been a physicist at IPP since 1981 and is responsible for the development of theoretical and numerical tools for the W7-X 
experiment. His research interest is kinetic theory.

Yuriy Turkin (PhD, Kharkov Institute of Physics and Technology, Ukraine, 1987) is a research associate in the theory group of Experimental Division E3 at the Max-Planck-Institut für Plasmaphysik (IPP), Greifswald, Germany, where he is responsible for the modeling of transport in W7-X. 